\title{
Genome Skimming is a low-cost and robust strategy to assemble complete mitochondrial genomes from ethanol preserved specimens in biodiversity studies
}

\author{
Bruna Trevisan ${ }^{\text {Equal first author, } 1}$, Daniel M. C. Alcantara ${ }^{\text {Equal first author, } 1 \text {, Denis Jacob Machado }}{ }^{1,2}$, Fernando P. L. Marques ${ }^{1}$, \\ Daniel J Lahr ${ }^{\text {Corresp. } 1}$ \\ 1 Department of Zoology, Institute of Biosciences, University of São Paulo, São Paulo, São Paulo, Brazil \\ 2 Department of Bioinformatics and Genomics / College of Computing and Informatics, University of North Carolina at Charlotte, Charlotte, NC, United \\ States \\ Corresponding Author: Daniel J Lahr \\ Email address: dlahr@ib.usp.br
}

Global loss of biodiversity is an ongoing process that concerns both local and global authorities. Studies of biodiversity mainly involve traditional methods using morphological characters and molecular protocols. However, conventional methods are a time consuming and resource demanding task. The development of high-throughput sequencing (HTS) techniques has reshaped the way we explore biodiversity and opened a path to new questions and novel empirical approaches. With the emergence of HTS, sequencing the complete mitochondrial genome became more accessible, and the number of genome sequences published has increased exponentially during the last decades. Despite the current state of knowledge about the potential of mitogenomics in phylogenetics, this is still a relatively under-explored area for a multitude of taxonomic groups, especially for those without commercial relevance, non-models organisms and with ancient DNA. Here we take the first step to assemble and annotate the genomes from HTS data using a new protocol of genome skimming which will offer an opportunity to extend the field of mitogenomics to under-studied organisms. We extracted genomic DNA from specimens preserved in ethanol. We used Nextera XT DNA to prepare indexed paired-end libraries since it is a powerful tool for working with diverse samples, requiring a low amount of input DNA. We sequenced the samples in two different Illumina platform (MiSeq or NextSeq 550). We trimmed raw reads, filtered and had their quality tested accordingly. We performed the assembly using a baiting and iterative mapping strategy, and the annotated the putative mitochondrion through a semi-automatic procedure. We applied the contiguity index to access the completeness of each new mitogenome. Our results reveal the efficiency of the proposed method to recover the whole mitogenomes of ancient DNA from non-model organisms even if there are gene rearrangement in the specimens. Our findings suggest the potential of combining the adequate platform and library to the Peer] reviewing PDF | (2019:01:34120:1:2:NEW 22 Jul 2019) 
genome skimming as an innovative approach, which opens a new range of possibilities of its use to obtain molecular data from organisms with different levels of preservation. 


\title{
Genome Skimming is a low-cost and robust strategy to assemble complete mitochondrial genomes from ethanol preserved specimens in biodiversity studies
}

\author{
Bruna Trevisan ${ }^{1 *}$, Daniel M. C. Alcantara ${ }^{1 *}$, Denis Jacob Machado², \\ Fernando P. L. Marques ${ }^{1}$, and Daniel J. G. Lahr ${ }^{1 * *}$ \\ ${ }^{1}$ Department of Zoology, Institute of Biosciences, University of São Paulo, São Paulo, \\ SP, Brazil \\ ${ }^{2}$ Department of Bioinformatics and Genomics, College of Computing and Informatics, \\ University of North Carolina at Charlotte, Charlotte, NC, USA \\ "These authors contributed equally to this work. \\ Corresponding author: \\ Senior Author** \\ Email address: dlahr@ib.usp.br
}

\begin{abstract}
16 ABSTRACT
Global loss of biodiversity is an ongoing process that concerns both local and global authorities. Studies of biodiversity mainly involve traditional methods using morphological characters and molecular protocols. However, conventional methods are a time consuming and resource demanding task. The development of high-throughput sequencing (HTS) techniques has reshaped the way we explore biodiversity and opened a path to new questions and novel empirical approaches. With the emergence of HTS, sequencing the complete mitochondrial genome became more accessible, and the number of genome sequences published has increased exponentially during the last decades. Despite the current state of knowledge about the potential of mitogenomics in phylogenetics, this is still a relatively under-explored area for a multitude of taxonomic groups, especially for those without commercial relevance, non-models organisms and with ancient DNA. Here we take the first step to assemble and annotate the genomes from HTS data using a new protocol of genome skimming which will offer an opportunity to extend the field of mitogenomics to under-studied organisms. We extracted genomic DNA from specimens preserved in ethanol. We used Nextera XT DNA to prepare indexed paired-end libraries since it is a powerful tool for working with diverse samples, requiring a low amount of input DNA. We sequenced the samples in two different Illumina platform (MiSeq or NextSeq 550). We trimmed raw reads, filtered and had their quality tested accordingly. We performed the assembly using a baiting and iterative mapping strategy, and the annotated the putative mitochondrion through a semi-automatic procedure. We applied the contiguity index to access the completeness of each new mitogenome. Our results reveal the efficiency of the proposed method to recover the whole mitogenomes of ancient DNA from non-model organisms even if there are gene rearrangement in the specimens. Our findings suggest the potential of combining the adequate platform and library to the genome skimming as an innovative approach, which opens a new range of possibilities of its use to obtain molecular data from organisms with different levels of preservation.
\end{abstract}

\section{INTRODUCTION}

Global loss of biodiversity is an ongoing process that concerns both local and global authorities (Vellend, 2017). Biodiversity loss impacts ecosystem functions, while additionally increasing the knowledge gaps and sampling biases (Asaad et al., 2017; Oliveira et al., 2017). Challenges including habitat loss, overexploitation, climate change, and invasive species are far from a solution (Corlett, 2017; Vellend, 
2017). Some advocate the use of natural history collections as a central tool for the study of biodiversity, especially for species that are becoming extinct or increasingly rare (Staats et al., 2013; Kanda et al., 2015). Studies of biodiversity mainly involve traditional methods using morphological characters and molecular protocols predominantly by PCR-based methods. However, conventional techniques are a time consuming and resource demanding tasks (Cameron, 014a; Yuan et al., 2016). Among molecular approaches, the PCR-based methods are often not successful in recovering genetic data of preserved organisms, due to the fragmented nature of old and poorly-preserved DNA (Heintzman et al., 2014; Timmermans et al., 2016). Also, the lack of genomic resources such as well-established and optimized molecular markers and primers to delimit target amplicons for closely related species may hamper PCR-based methods for non-model organisms (Ekblom and Galindo, 2011; Tilak et al., 2015; Matos-Maraví et al., 2018).

The development of high-throughput sequencing (HTS) techniques has reshaped the way we explore biodiversity and opened a path to new questions and novel empirical approaches (Dodsworth, 2015; Linard et al., 2015; Porter and Hajibabaei, 2018). The use of low-coverage and cost-effective genome-skimming, also known as whole-genome shotgun sequencing (WGS), is one of these techniques. This particular method consists of sequencing the whole genome of an individual at low nuclear genome coverage. The process provides an extensive data set, capable of recovering high-copy fractions of total genomic DNA (organellar genomes, nuclear ribosomal DNA, and other multi-copy elements) through random shearing and inexpensive multiplexing (Berger et al., 2017; Matos-Maraví et al., 2018). The technique is potentially efficient for old museum material and ethanol-preserved specimens (Staats et al., 2013; Maddison and Cooper, 2014; Linard et al., 2016; Grandjean et al., 2017). Despite recent efforts to obtain DNA sequences through HTS protocols from museum specimens (McCormack et al., 2017; Dabney et al., 2013; McCormack et al., 2016) and ethanol material (do Amaral et al., 2015; Brabec et al., 2015, 2016; Hartikainen et al., 2016; Vanhove et al., 2018), the potential use of genome skimming for this purpose remains unexplored. There is a variety of suitable HTS sequencing platforms and library options to choose from to use in combination with genome skimming, including PCR-based libraries and PCR-free libraries that are less-error prone but require higher input DNA $(\leq 1 \mu g)$ (Twyford and Ness, 2017). Knowing that the preservation level of biological samples can influence the quality of sequencing, it is important to consider the amount of input DNA available. Since researchers often preserve specimens of invertebrates in ethanol, specific protocols are required to obtain high-quality data from low quality or quantity DNA extracts (Tilak et al., 2015; Linard et al., 2016). Therefore, combining the adequate platform and library to the genome skimming technique is an innovative approach, which could overcome most of the limitations highlighted above, opening a new range of possibilities of its use for obtaining molecular data.

The mitogenome has been used as a molecular marker in a great variety of studies (e.g. ecology, evolution, phylogeography and phylogenetics at multiple taxonomic levels; see Avise et al., 1987; Le et al., 2000; Zarowiecki et al., 2007; Avise, 2012; Li et al., 2017). Its popular use throughout those areas could be attributed to its particularity as maternal inheritance, high copy-number, lack of recombination and higher mutation rate when compared to other markers (Ballard and Whitlock, 2004; Hahn et al., 2013; Yuan et al., 2016; Li et al., 2017). Animal mitochondrial genomes are generally uniform across metazoan groups (Park et al., 2006; Tan et al., 2017): a circular, double-stranded DNA molecule, ranging from 15-20 kb in size, containing circa 37 genes (i.e. two mitochondrially encoded ribosomal RNAs [rDNA], 13 protein-coding genes [PCG] and 22 transfer RNA genes [tRNA]) (Park et al., 2006; Castellana et al., 2011; Bernt et al., 2013; Tan et al., 2017). This set of attributes provide to mitogenomes a wide spectrum of informational content, which can be used to answer many biodiversity questions.

Despite the potential of mitogenomes in solving biodiversity questions, the great majority of the studies only targeted a small fraction of this genome. The prevalence of partial sequences of the mitochondrially encoded ribosomal RNAs MT-RNR1 and MT-RNR2 (12S and 16S, respectively), Cytochrome B (MTCYB) and Cytochrome C Oxidase I (MT-CO1) in many studies can be credited to the existence of "universal primers" that amplified these regions for a whole spectrum of non-model Metazoa taxa. Hence, it is not uncommon to find studies addressing phylogenetic relationships at different levels of divergence-including the interest on detecting cryptic species-based on these markers (von NickischRosenegk et al., 1999; Zehnder and Mariaux, 1999; Hu et al., 2005; Wickström et al., 2005; Littlewood et al., 2008; Brabec et al., 2016; Vanhove et al., 2018, to cite a few). However, to date HTS provided the means of sequencing the complete mitochondrial genome in reasonable time and at relative low cost. As a result, the number of genome sequences published has increased exponentially during the last decades (Park et al., 2006; Hahn et al., 2013; Tan et al., 2017; do Amaral et al., 2015; Vanhove et al., 2018). With 
the increase of studies using complete mitogenomes, several authors have recognized the virtues of a greater amount of nucleotide sequence data for inferring robust phylogenies in many taxonomic groups such as mammals (Arnason et al., 2002; Campbell and Lapointe, 2011), birds (Pacheco et al., 2011), insects (Cameron, 2014), and flatworms (Brabec et al., 2015, 2016; Maldonado et al., 2017; Vanhove et al., 2018). In addition, we can also relate the power of resolution of the mitogenome to its genome-level characteristics such as gene arrangements and the positions of mobile genetic elements, which are good alternatives to resolve deeper phylogenetic questions (Waeschenbach et al., 2012; Guo, 2015; Li et al., 2017).

In spite of its undeniable informational content, whole mitogenomes are still relatively under explored in phylogenetic studies for a multitude of taxonomic groups, especially for those without commercial relevance, non-model organisms, and ancient DNA (Littlewood et al., 2008; Maldonado et al., 2017). The majority of these groups still have poorly understood phylogenetic histories. Two examples such groups are cestodes in the family Rhinebothriidae and dipterans of the family Streblidae. Rhinebothriideans are exclusively endoparasites of batoid elasmobranchs, while Streblids are highly specialized ectoparasites of bats (Dick et al., 2016; Ruhnke et al., 2017). Both groups have intricate historical associations with their hosts; which are of scientific interest of evolutionary biologists engaged in understanding how historical association events shaped the ecology, patterns of association and evolution of these host/parasite systems (Wenzel et al., 1966; Brooks et al., 981b; Dick and Patterson, 2006; Tello et al., 2008; Marques and Caira, 2016).

However, despite the efforts to reconstruct the phylogenies using fragments of mtDNA (or, in the case of rhinebothriideans, only pieces of rRNA genes), their internal relationships remain poorly understood. We could attribute this lack of understanding to a number of factors including the difficulty in extracting DNA from fixed organisms, the low resolution of those markers, and the limited availability of sequenced samples (Dittmar et al., 2006; Petersen et al., 2007; Caira et al., 2014; Ruhnke et al., 2015; Trevisan et al., 2017). Thus, mitogenomics carries the potential to resolve the phylogenetic history in those groups. Here we take the first step to assemble and annotate the genomes from HTS data using a new genome skimming protocol, revealing an opportunity to extend the field of mitogenomics to under-studied organisms.

\section{METHODS}

\section{Taxon sampling}

We fixed all samples in $96 \%$ ethanol and stored them at $-20{ }^{\circ} \mathrm{C}$ until we performed the genomic DNA extractions. Hologenophores (sensu Pleijel et al. 2008) from Streblidae and Rhinebothriidae were deposited at MZUSP (Museu de Zoologia da Universidade de São Paulo, Universidade de São Paulo, São Paulo, SP, Brazil). We collected the batflies in Brazil, Paradyschiria parvula Falcoz, 1931 (Voucher No. XXX) from Base de Estudos do Pantanal, Passo do Lontra, Corumbá, Mato Grosso do Sul (19³4'48.0'S, 57 01'15.1'W) in 2013 and Paratrichobius longicrus (Miranda Ribeiro, 1907) (Voucher No. XXX) from Núcleo Pedra Grande, Parque Estadual Cantareira, São Paulo, São Paulo (2326'10.9”'S, $\left.46^{\circ} 38^{\prime} 07.8^{\prime \prime} \mathrm{W}\right)$ in 2017 . We collected the hosts following the permit guidelines issued by Sistema de Autorização e Informação em Biodiversidade - SISBIO (5184-1, issued in 2013, Brazil to Gustavo Graciolli from Universidade Federal do Mato grosso do Sul to sample Paradyschiria parvula and by SISBIO and by Secretaria do meio Ambiente - SMA to sample Paratrichobius longicrus (55242-1 and 260108-008.107/2016, respectively) both issued in 2016, Brazil to Daniel Maximo Corrêa de Alcantara. Under those permits, we captured these hosts using mist nets, opened at ground level in trails and other locations near to bodies of water during 6 hours after sunset, and checked them every 30 min. We examined the bats for ectoparasites manually or with the aid of tweezers.

We obtained Anindobothrium anacolum Marques, Brooks \& Lasso, 2001 (Voucher MZUSP 7968) and Rhinebothrium reydai Trevisan \& Marques, 2017 (Voucher MZUSP 7969) from the spiral intestines of the stingray Styracura schmardae from Trinidad \& Tobago (Maracas, San Juan-Laventille, $10^{\circ} 45^{\prime} \mathrm{N}$, $61^{\circ} 26^{\prime} \mathrm{W}$ ) in 2014 and from Panama (Almirante, Bocas del Toro, $9^{\circ} 17^{\prime} \mathrm{N}, 82^{\circ} 20^{\prime} \mathrm{W}$ ) in 2015 , respectively. We collected these hosts using spears following the permit guidelines issued by the Ministry of Food Production-Fisheries Division (issued in September 2014, Trinidad \& Tobago to F.P.L. Marques) and by the Autoridad Nacional del Ambiente-ANAM (SE/A-101-14, issued in December 2014, Panama to F.P.L. Marques), respectively. Further details on the collection of hosts and specimens preparation is available in Trevisan et al. (2017) and Marques and Reyda (2015). 


\section{DNA extraction}

For Streblidae, we extracted DNA using the Qiagen DNeasy Blood \& Tissue Kit (Qiagen). Since it is common the abdomen of Streblidae contain the host's blood, we separated the thorax of each specimen from the abdomen, and only the thorax with the head and legs were used to avoid contamination. The DNA was eluted in $200 \mu l$ of a buffer solution, repeating the elution step twice with the addition of $100 \mu l$ each time for increased DNA yield. After extraction, we stored the thorax with the head and legs in ethanol, together with the specimen abdomen, and cataloged as specimen vouchers.

For Rhinebothriidae, we extracted the DNA from the middle portion of the strobila of each specimen, which was removed and allowed to air dry for about 5 minutes at room temperature. We extracted total genomic DNA using Agencourt DNAdvance - Nucleic Acid Isolation Kit (Beckman Coulter) following the manufacturer's instructions. We prepared scolices and posterior portions of strobila from specimens used in molecular analyses as whole mounts following traditional protocols (Trevisan et al., 2017).

We employed standard precautions to minimize contamination throughout, such as using exclusive pipettes with filter tips and bleaching all the instruments used in DNA extraction. We measured the purity and amount of DNA extractions using a NanoDrop 2000 spectrophotometer (Thermo Fisher Scientific) and Qubit 2.0 Fluorometer using Qubit high sensitivity dsDNA assays (Life Technologies).

\section{Library preparation and sequencing}

We used Nextera XT DNA Library Preparation Kit (Illumina) to prepare indexed paired-end (PE) libraries according to the manufacturer's protocol. We chose Nextera XT to prepare the libraries because the fabricator optimized the protocol for $1 n g(5 \times 0.2 n g / \mu l)$ of input DNA in total. The low amount of input DNA provides a powerful tool for working with a variety of samples that yield either small or copious amounts of tissue. This library is also likely to be suitable for DNA extractions from samples of model and non-model taxa with different ages of fixation, especially for small genomes $(\leq 5 M b)$, PCR amplicons, and plasmids.

Before starting libraries preparation, we diluted DNA extracts in Milli-Q water to $0.2 n g / \mu l$, after which we checked the concentration in a Qubit 2.0 Fluorometer. We used a low-cost method to determine the quality and size of the sequencing libraries, as performed in Kang et al. (2017). The technique consists of PCR amplification of the library, using Illumina adapter primers, checking amplicons for quality and size by standard agarose gel electrophoresis. We prepared a PCR master mix with the appropriate volume for each sample, containing $5 \mu l$ of KAPA Taq ReadyMix PCR Kit, $3 \mu l$ of Milli-Q water, $0,5 \mu l$ of Illumina forward primer $(10 \mu M), 0,5 \mu l$ of Illumina reverse primer $(10 \mu M)$ and $1 \mu l$ of DNA template. Then, we run PCR using the following protocol: $45 \mathrm{sec}$ of initial denaturation at $98{ }^{\circ} \mathrm{C} ; 20$ cycles of 25 sec of denaturation at $98{ }^{\circ} \mathrm{C}, 30 \mathrm{sec}$ of annealing at $47^{\circ} \mathrm{C}$, and $1 \mathrm{~min}$ and $30 \mathrm{sec}$ of extension at $72{ }^{\circ} \mathrm{C}$; 3 min of final extension at $72{ }^{\circ} \mathrm{C}$; and hold at $4{ }^{\circ} \mathrm{C}$. Subsequently, we examined these PCR products in $1.8 \mathrm{x}$ TBE agarose gel electrophoresis. We determined the sequencing library concentrations on Qubit 2.0 Fluorometer using Qubit high sensitivity dsDNA. Library normalization was done manually, diluting libraries to the same concentration $(4 n M)$ before volumetric pooling.

We sequenced the samples of Rhinebothriidae and Streblidae in two different Illumina platforms. Samples of Streblidae were sequenced alone using an Illumina MiSeq System, with a Reagent Kit v3 to generate PE reads of 300bp. Since it is possible to sequence 24-30 million reads with the specifications used to run the Illumina MiSeq System, we pooled up to two DNA libraries. The samples of Rhinebothriidae were sequenced using an Illumina NextSeq 550 System, with a High-Output Kit to generate PE reads of $150 \mathrm{bp}$. This system can sequence up to 800 million reads with these specifications, allowing us to pool up to 35-40 DNA libraries. Thereby, each lane of the Illumina NextSeq 550 also received 33 additional libraries. We based the calculation of the number of reads required per sample to recover the complete mitochondrial genome on Richter et al. (2015). We performed all DNA sequencing in the Core Facility for Scientific Research—University of São Paulo (USP) (CEFAP-USP).

\section{Computational resources}

We executed in silico procedures using "ACE", an SGI rackable computer cluster housed in the Museum of Zoology of the University of São Paulo. Select servers had four $2.3 \mathrm{GHz}$ Operon CPUs with 16 cores each and 256 or 516 GB of memory. The software environment in ACE consists of a SUSE Linux Enterprise Server with SGI Performance Suite, SGI Management Center and PBS Pro Job Scheduler. We were able to reconstruct each genome using a single core and ca. 20 GB of memory. 


\section{Manuscript to be reviewed}

\section{Quality control and mitogenome assembly}

We pre-processed the raw reads from each pair using a series of UNIX commands. We trimmed and filtered the sequences using the HTQC toolkit (Yang et al., 2013) a home-made Python script (selectTiles.py, see Machado et al. 2016) that automates tiles selection. We evaluated the quality of filtered reads with FASTQC (Andrew, 2010). The assembly protocol received only filtered PE reads. We described the complete quality control protocol below and the step-by-step procedures are given in Machado et al. (2016, Appendix S1).

We performed the sequence assembly using a baiting and iterative mapping strategy based on MIRA v4.0 Chevreux et al. (1999) and a modified version of MITOBIM.PL v1.6 (Hahn et al., 2013), following the guidelines described in Machado et al. (2016, Appendix S2). We applied the same search parameters to every assembly but used different baits depending on the class of the specimen. The reference mitogenome sequence of the house fly (Musca domestica L., GenBank Accession Number KM200723) was the bait for all streblids, and the reference mitogenome sequence of the beef tapeworm (Taenia saginata Goeze 1782, GenBank Accession Number NC_009938) was the bait for the assembly of cestodes. Finally, we inferred the completeness of each new putative mitogenome (i.e., sequence circularization) using the AWA program and the contiguity index statistics described in Machado et al. 2018; the AWA beta version is available at https://gitlab.com/MachadoDJ/awa.

We mapped the raw sequence reads back to the putative mitogenome selected by AWA with Bowtie2 v2.2.6 (Langmead and Salzberg, 2012) using the local alignment algorithm and the highest sensitivity setting. We set the threshold for base calling on the consensus sequence to bases that match at least $99 \%$ of the sequences, with a minimum coverage per position of 3 sequences.

\section{Mitogenome annotation}

We parsed the assemblies in CAF format using a home-made Python script (parseCaf.py; see Machado et al. 2016) to extract DNA data and evaluate the coverage and quality of each mtDNA element. Preliminary de novo mitogenome annotation used the mitochondrial genome annotation server MITOS2 (Bernt et al., 2013, available at http://mitos2.bioinf.uni-leipzig.de), changing the genetic code accordingly (transl_table=9 for flatworm, transl_table $=4$ for insects).

We applied three different strategies independently to corroborate the annotations of coding genes. We used the BLAST (Altschul et al., 1990) to search a selected database of mitochondrial peptides from UniProt/ Swiss-Prot (The UniProt Consortium, 2016; UniProt resources are available at http://www.uniprot.org). We executed a second comparison between reference amino acid sequences and the new nucleotide sequences with GeneWise (Birney et al., 2004). Finally, we also applied TransDecoder (see Hahn et al. 2013; the program is available at https://github.com/TransDecoder) to identify candidate coding regions and compare the outputs from these programs to propose the final annotations.

We performed additional search and validation of tRNA sequences using ARWEN (Laslett and Canbäck, 2007) and tRNAscan-SE (Lowe and Eddy, 1997; Schattner et al., 2005).We confirmed and edited manually the automated annotation by comparison to published reference mitogenomes of flies and tapeworms. We annotated the control region (CR) with sequence similarity searches in BLAST using default parameters.

Annotation of cestode mitogenomes was less straightforward compared to streblid organelles and required a more complex strategy. This was needed because some software mentioned above and used for annotation have not yet fully implemented the traditional codon table for flatworms (transl_table=9). Besides, nucleotide and amino acid reference sequences were not available. These limitations reduced the efficiency of the annotations of flatworm sequences, resulting in some wrong start or end positions or missing genes. Although they did not impede our semi-automatic annotation strategy, the procedure was very time-consuming and required manual curation. Fig. 1 shows a summary of the workflow of the protocol we proposed in this study.

\section{RESULTS}

\section{DNA extraction, library preparation and genome sequence}

We obtained sufficient DNA quantities for all samples (Table 1), but we required some additional protocols to achieve the initial concentration for library preparation. We diluted the samples from A. anacolum and $R$. reydai because they contained a DNA concentration higher than initially required. The DNA concentration obtained for $P$. longicrus was closer to the amount necessary to prepare the library, and 
Figure 1. Schematic workflow of the new protocol proposed in this study.

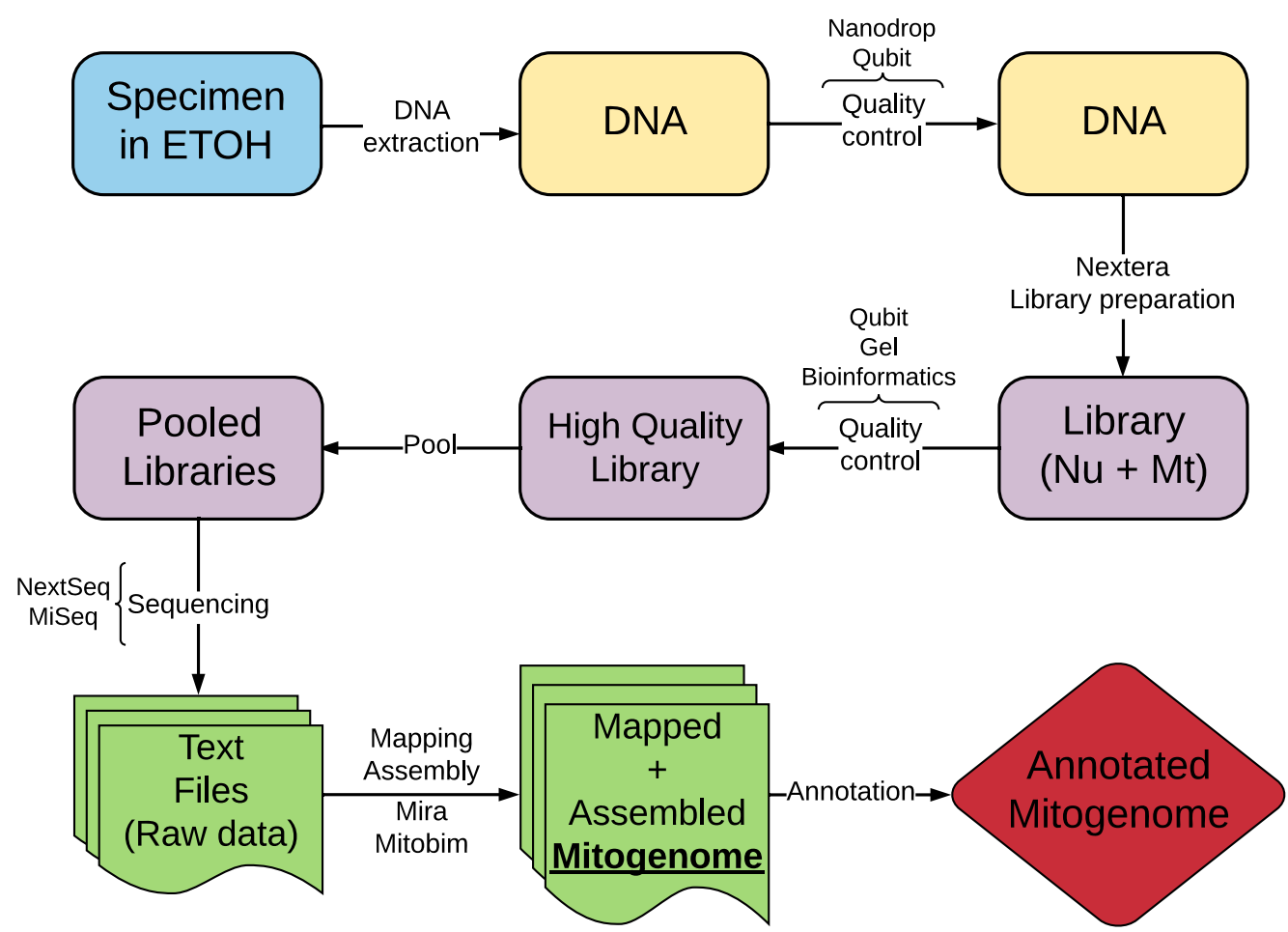

therefore its DNA was not diluted. In the case of Paradyschiria parvula, the DNA concentration was too low $(\leq 0,50 \mathrm{ng} / \mathrm{ml})$. In this particular case, we concentrated the extracted DNA using Agencourt AMPure XP and eluting the DNA in $15 \mathrm{ml}$. This protocol allowed us to reach the concentration close to that necessary to start the library preparation, without the need for dilution (Table 1). The sequence on Illumina MiSeq resulted in a total of $13.98 \mathrm{mi}$ PE reads for $P$. parvula and $16.82 \mathrm{mi} \mathrm{PE}$ reads for $P$. longicrus. For Rhinebothriidae, the sequence on Illumina NextSeq resulted in a total of $11.81 \mathrm{mi}$ PE reads for A. anacolum and $7.04 \mathrm{mi} \mathrm{PE}$ reads for $R$. reydai.

Table 1. Concentration of DNA in the extraction and library preparation from the specimens included in this study. The measurements were performed in Qubit 2.0 Fluorometer and the values are given in $n g / \mu l$. A, after DNA extraction; B, after using Agencourt AMPure XP; Dilution, after diluting the DNA extracted to start the library preparation; Final, after library preparation with Nextera XT.

\begin{tabular}{lcccccc}
\hline \multirow{2}{*}{ Species } & \multicolumn{2}{c}{ Extraction } & & \multicolumn{2}{c}{ Library Prep } \\
\cline { 2 - 3 } \cline { 5 - 6 } & $\mathbf{A}$ & $\mathbf{B}$ & & Dilution & Final \\
\hline Paradyschiria parvula & $\leq 0.1$ & 0.184 & & - & 2.12 \\
Paratrichobius longicrus & 0.354 & - & & - & 3.34 \\
Anindobothrium anacolum & 18.6 & - & & 0.208 & 14.2 \\
Rhinebothrium reydai & 9.08 & - & & 0.260 & 12.9 \\
\hline
\end{tabular}

\section{Mitogenome assembly}


was high, 1,749.6 for $P$. parvula and 6,355.0 for P. longicrus. Their mitogenome length conforms with those found in other Diptera, typically $14-19 \mathrm{~kb}$ (Li et al., 2015). The total number of mapped sequences for $P$. parvula was 96,404 whereas $P$. longicrus shown a higher value of 695,060. However, circularity tests of the mitogenome of $P$. parvula presented a higher average coverage, contiguity, and score than $P$. longicrus (329 vs. $37.3,322.3$ vs. 36.3 and -1.43 vs. -6.79 , respectively) (Table 2). The lower score of $P$. longicrus is bound to be affected by the ambiguous nucleotides obtained from our conservative approach to base calling (detailed below).

The mitogenome sequences obtained for specimens of Rhinebothriidae, A. anacolum (GenBank accession no. MK887326) and $R$. reydai (GenBank accession no. MK896864), were 13,693 bp and $13,506 \mathrm{bp}$ in length with 743.4 and 258.5 coverage, respectively. Their mitogenome size follows the pattern observed previously for Cestodes (13-15kb) (Li et al., 2017). The total number of mapped sequences for $A$. anacolum was 83,969 and $R$. reydai shown a smaller value of 28,583 . We observed the opposite for the contiguity index in which $A$. anacolum had a lower value in comparison to $R$. reydai (153.2 vs. 313.8, respectively). The GC content and quality of the sequences was similar for both species (30.4\% vs. $35.8 \%$ and 34.0 vs. 32.4, respectively). Overall the scores $A$. anacolum and $R$. reydai were close to 0 , which should be considered as another good indicator of quality (non-ambiguity) (Table 2 ).

Table 2. General assemble statistics. Q1 and Q3 indicate $1^{\text {st }}$ and $3^{\text {rd }}$ quartiles for coverage, respectively. Asterisks indicate indexes calculated during circularization tests in AWA (Machado et al. 2018), using the 50 nucleotides flanking each end of the putative mitochondrion contig.

\begin{tabular}{|c|c|c|c|c|c|c|c|c|}
\hline \multirow[b]{2}{*}{ Species } & \multirow[b]{2}{*}{ Length } & \multirow[b]{2}{*}{ GC\% } & \multirow[b]{2}{*}{$\begin{array}{c}\text { No. of } \\
\text { Sequences }\end{array}$} & \multirow[b]{2}{*}{$\begin{array}{c}\text { Avg. Coverage } \\
\text { (Q1 : Q3) }\end{array}$} & \multicolumn{4}{|c|}{ AWA } \\
\hline & & & & & $\begin{array}{c}\text { Avg. } \\
\text { Coverage* }\end{array}$ & $\begin{array}{c}\text { Avg. } \\
\text { Contiguity* }\end{array}$ & $\begin{array}{c}\text { Avg. } \\
\text { Quality* }\end{array}$ & $\begin{array}{c}\text { Bowtie2 } \\
\text { Score* }\end{array}$ \\
\hline P. parvula & 14,588 & 21.4 & 96,404 & $\begin{array}{c}1,749.6 \\
(841: 2,133)\end{array}$ & 329 & 322.3 & 35.8 & -1.43 \\
\hline P. longicrus & 16,296 & 17.9 & 695,060 & $\begin{array}{c}6,355.0 \\
(5,506: 7,945)\end{array}$ & 37.3 & 36.3 & 36.0 & -6.79 \\
\hline A. anacolum & 13,693 & 30.4 & 83,969 & $\begin{array}{c}743.4 \\
(526: 962)\end{array}$ & 157.3 & 153.2 & 34.0 & -0.92 \\
\hline R. reydai & 13,506 & 35.8 & 28,583 & $\begin{array}{c}258.5 \\
(216: 305.3)\end{array}$ & 318.9 & 313.8 & 32.4 & -2.45 \\
\hline
\end{tabular}

\section{Mitogenome organization and structure \\ Streblidae}

The mitogenome of both species contains 37 genes, including 13 PCGs, 22 tRNA genes, two rRNA genes and the CR. We recovered $71 \mathrm{bp}$ of the CR for P. parvula and 1,579 bp for P. longicrus. For both species, twenty-three genes are encoded on the majority strand (14 tRNAs and nine PCGs), while the minority strand encodes the remaining 14 genes (eight tRNAs, the two ribosomal RNAs, and four PCGs) (Fig. 2). We must note a bias in the nucleotide composition of the mitogenome toward A and T, with a GC content of $21.4 \%$ for P. parvula and $17.9 \%$ for P. longicrus (Table 2). Except for MT-CO1, each of the thirteen PCGs had the canonical start codon ATT (encoding Ile) or ATG (encoding Met). We identified the MT-CO1 start codon as TCG (encoding Ser). We found incomplete stop codons (T) only in MT-ND4 for $P$. parvula. All other PCGs have the complete stop codons TAA or TAG in both species, being TAG found only for MT-CYB. We also found several stop codons TGA within all PCGs.

\section{Rhinebothriidae}

Each mitogenome contains 36 genes, including 12 protein-coding genes (MT-ATP6, MT-CO1-3, MT-CYB, MT-ND1-6 and MT-ND4L), 22 transfer RNA genes (tRNA), two ribosomal RNA genes (RNR1-2) and one CR. As previously reported for other Neodermata mitogenomes, the specimens from Rhinebothriidae also lacks the ATP8 gene, which is found in other metazoan mitogenomes (Le et al., 2002; Guo, 2016; Zhao et al., 2016; Li et al., 2017; Egger et al., 2017). We noticed that the nucleotide composition of the mitogenome is biased towards A and T, with a GC content of $35.8 \%$ for $R$. reydai and $30.4 \%$ for $A$. anacolum (Table 2). All genes are encoded in the same strand and transcribe from the same direction. The gene order in A. anacolum and $R$. reydai follows the typical organization of cestodes, except by the 
rearrangements of some tRNA genes and by the number of non-coding regions (NCR) (i.e. one). Despite the slightly differences, these results reinforces previous evidences that cestode gene order is extremely conserved (Nakao et al., 2003; Li et al., 2017; Zhang et al., 2017). The reader should note that annotations described here for the mitogenomes of flatworms derive from a semi-automatic and manually curated annotation protocol but, given the specific challenges involved in annotating this mitogenomes, we are currently preparing a pipeline dedicated to their annotation, which we will present elsewhere.

Figure 2. Graphical representation of the mitogenomes of A. Anindobothrium anacolum, B.

Rhinebothrium reydai, C. Paradyschiria parvula, and D. Paratrichobius longicrus. Grey: control region; yellow: CDS; green: genes; red: rRNA; pink: tRNA.
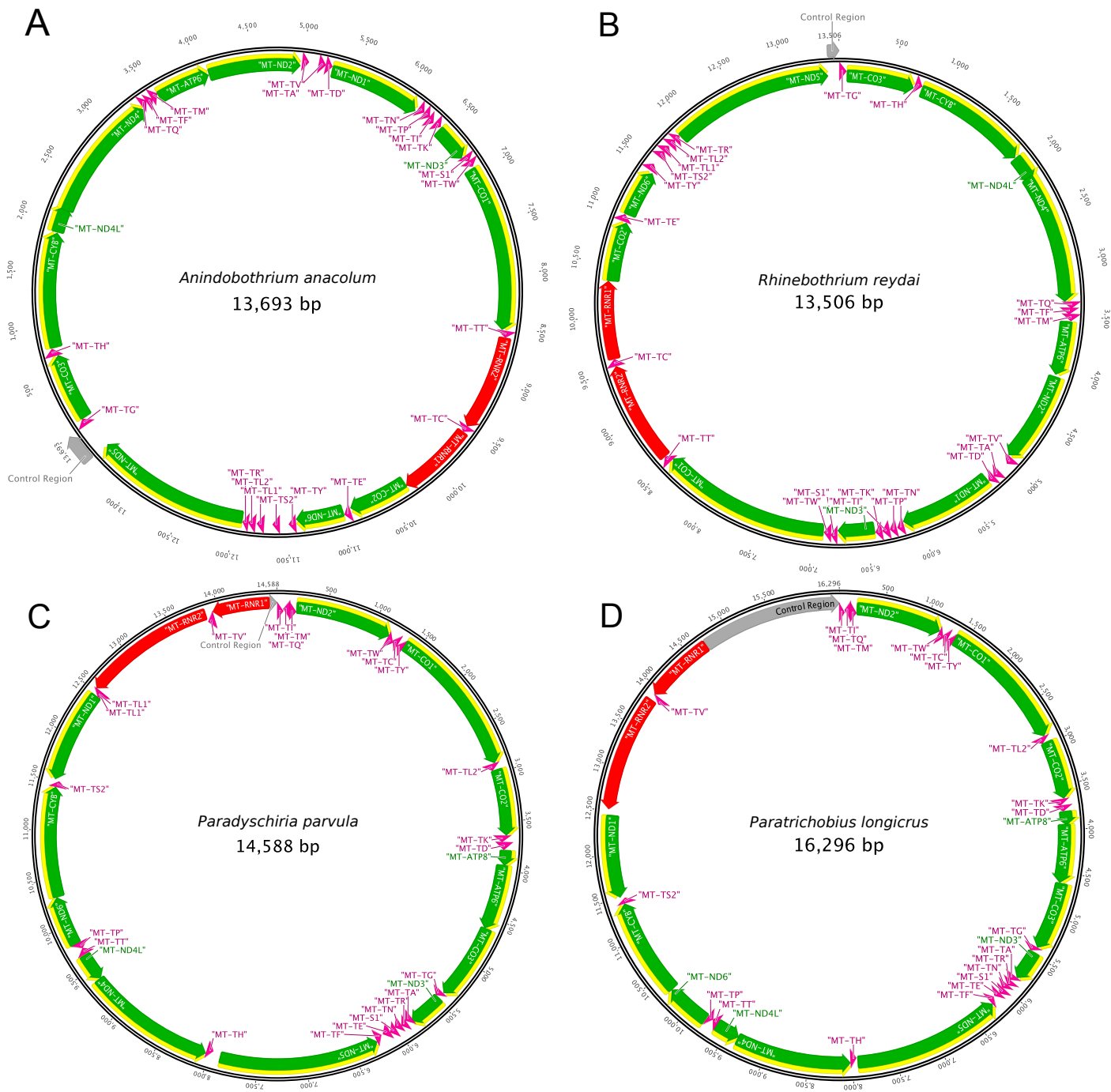

\section{DISCUSSION}

\section{Coverage variation}

The overall quality and alignment score of the assembly of the four mitogenomes indicate that even with relatively lower coverage values, it was possible to recover the mitochondrial genome with this protocol. Given our conservative approach towards base calling, we left some ambiguous nucleotides in six tRNAs, two rRNAs, 11 PCGs and the CR of the mitogenome of $P$. longicrus. However, the number of these ambiguities is within our expectations given the procedures described here and the expected variation in the percentage of mitochondrial among different libraries. 


\section{Manuscript to be reviewed}

As expected, there was a wide variation on the number of reads mapped to each mitochondrial genome. The ratio between the number of mitochondria and the genome size of the organism influences the number of mitochondrial reads in a library (Richter et al., 2015). In tunicates, for instance, a lower proportion of mitochondria to nuclei in tissues is correlated to reduced numbers of mitochondrial reads (Tilak et al., 2015). The mitogenome coverage may even correlate negatively with the amount of DNA used for sequencing, since higher amounts of DNA can increase the chance of introducing more nuclear reads into the library, reducing the number of mitochondrial reads. Furthermore, the number of multiplexed species per sequencing can also influence the number of mitochondrial reads sequenced and the average coverage (Richter et al., 2015; Tilak et al., 2015). Therefore, the number of recovered mitochondrial reads in different genome-skimming experiments can vary.

The quality of the assembly around the $50 \mathrm{bp}$ flanking each end of the putative mitogenomes is also presumed to vary. For the assembly of $P$. longicrus mitogenome, which has the lowest coverage and contiguity score in AWA, our analysis found $k$-mer lengths that obtained an average coverage higher than that presented in Table 2 (up to 207.73x). However, the identity and quality of these alignments were much lower, and therefore they were not selected by AWA software based on the combined criterion of read coverage, read contiguity, average quality, and alignment scores.

\section{Streblidae mitogenome order rearrangements}

Compard to other dipterans, the gene order and sizes follows the typical organization ported for this group (Nelson et al., 2012; Cameron, 2014; Li et al., 2015; Pu et al., 2017). Among flies, ATG (Met) and ATT (Ile) are frequently found as start codons (Li et al., 2015), which is congruent with our results. On the other hand, start codons for MT-CO1 are usually considered non-canonical in holometabolans, though TCG is widely reported for MT-CO1 in Diptera (Cameron et al., 2007; Nelson et al., 2012). The stop codon most commonly found in Diptera is TAA. However, partial stop codon T has been reported in many insect mitogenomes and is completed to a full TAA stop codon via post-transcriptional polyadenylation (Li et al., 2015). Moreover, some authors have already reported that translation termination might reassign the codon UGA to code for tryptophan or cysteine (Alkalaeva and Mikhailova, 2017), which may be the case in this study. Even though the CR can be variable in both size and nucleotide composition, the difference in size between the two species of Streblidae is striking. More study are important to investigate such difference, since cases of duplication in the CR are known in different groups, including insects (Cameron, 2014; Yan et al., 2012).

Although Diptera is one of the most extensively sequenced orders within Insecta, the number of complete mitogenomes is low given its diversity (Liu et al., 2017; Narayanan Kutty et al., 2019). Streblidae belongs to the Calyptratae clade, one of the most species-rich group within Diptera with over 22,000 species. Calyptratae is divided into three superfamilies: Hippoboscoidea (to which Streblidae belongs), Muscoidea and Oestroidea (Narayanan Kutty et al., 2019). Currently, 231 mitochondrial genome sequences of Diptera are included in the NCBI GenBank Organelle Genome Resources (see also Benson et al., 2008). However, 51 of these mitochondrial genomes are from Calyptrates, while 41 are from Oestroidea, nine from Muscoidea and only one from Hippoboscoidea. It is clear that the Calyptratae clade is poorly represented, and the two new sequences will allow a significant gain not only for the family in question but also at many levels in insect systematics.

\section{Cestode mitogenome order rearrangements}

The most recent and comprehensive study on the diversity of mitogenomes in cestodes was published by $\mathrm{Li}$ et al. (2017). In this study, the authors included 54 mitogenomes representing 5 of the 18 orders presently recognized for cestodes (following Caira et al., 2014). The study included members of Caryophyllidea, Diphyllobothriidea, Bothriocephalidea, Onchoproteocephalidea and Cyclophyllidea; however, the latter order comprised $75 \%$ (41) of the species included in the study. Hence, this is the first attempt to document the diversity of mitogenomes within this group.

The authors considered that mitogenome gene order is extremely conserved in cestodes. Their assertion was based on the observation that all mitogenomes studied could be attributed to 4 categories based on the arrangements coding regions and tRNAs. According to Li et al. (2017), caryophyllideans possesses a Category I mitogenome from which Category II derived by a transposition event in which the region $t R N A^{\operatorname{Leu}(C U N)}-t R N A^{\operatorname{Ser}(U C N)}-t R N A^{\operatorname{Leu}(U U R)}$ translocated to the 3' end end of the four genes $\operatorname{cox} 2-t R N A^{G l u}-N a d 6-t R N A^{T y r}$. Following, two other categories would have derived from Category II; one by a tandem duplication and random loss (TDRL) event that generated the region $t R N A^{\operatorname{Leu}(C U N)}-$ 
$t R N A^{\operatorname{Leu}(U U R)}-t R N A^{T y r}-t R N A^{\operatorname{Ser}(U C N)}$ —Category III; and the other by a simple transposition event generating the region $t R N A^{\operatorname{Ser}(U C N)}-t R N A^{\operatorname{Leu}(C U N)}$-Category IV.

Although Li et al.'s study should be considered a preliminary account of the diversity of mitogenomes within cestodes, there appears to be some phylogenetic congruence among the patterns of gene rearrangements that can generate some predictions for future studies. The transposition that characterizes their Category II was found in all polyzooic orders included in the study (i.e., Diphyllobothriidea, Bothriocephalidea, Onchoproteocephalidea and Cyclophyllidea). The hypothesized TDRL resulting in the region $t R N A^{\operatorname{Leu}(C U N)}-t R N A^{\operatorname{Leu}(U U R)}-t R N A^{T y r}-t R N A^{\operatorname{Ser}(U C N)}$ found in three species of Schyzocotyle could be a putative synapomorphy for the genus, if not for the order Bothriocephalidea. Finally, the transposition leading to the region $t R N A^{\operatorname{Ser}(U C N)}-t R N A^{\operatorname{Leu}(C U N)}$ could be a synapomorphy for acetabutate cestodes (sensu Caira et al, 2014) - although it seems to be reverted to the ancestral state $\left(t R N A^{\operatorname{Leu}(C U N)}-t R N A^{\operatorname{Ser}(U C N)}\right)$ in taeniids.

Our results confirm some of these predictions. Rhinebothriideans are considered to have derived after bothriocephalideans and the order is considered to be sister to a large clade comprised by 6 orders including Onchoproteocephalidea and Cyclophyllidea (see Caira et al., 2014). As predicted the mitogenomes of Anindobothrium anacolum and Rhinebothrium reydai share the region $t R N A^{\operatorname{Ser}(U C N)}-t R N A^{\operatorname{Leu}(C U N)}$ found in acetabutate cestodes, with the exception of teaniids (i.e. Category IV). However, contrary to most mitogenomes known to date for cestodes, our results indicate that rhinebothriideans have only one non-coding regions (NCRs), an attribute also found in the taeniid Hydatigera taeniformis (Batsch, 1786) (Li et al., 2017). However, taeniids seem to have reverted to the ancestral gene arrangement of Category II.

Li et al. (2017) acknowledged the possibility that the diversity of arrangements is underestimated given the taxonomic representativity of their dataset. In fact, when NCRs are taken into account, we found that there are few arrangements that were not considered by the authors. For instance, the bothriocephalid Schyzocotyle acheilognathi posses a third NCR not found in any other cestode. Within Category IV, there are at least 7 distinct arrangements if you consider the position of NCRs; $A$. anacolum and $R$. reydai yet have a different one. We predict that as we compile mitogenomes for cestodes, we will have a better understanding of the rearrangement events that explain the diversity in the group. To achieve this goal, it would be desirable to have representatives of all major lineages of cestodes and access variability in different taxonomic levels. We also think that special attention should be given to homology statements of NCRs since we already have an indication that the number of NCRs differ within the groups (see Li et al., 2017). Finally, we think that once we achieve the goals above and contextualize mitogenome rearrangements within a phylogenetic context we might uncover new synapomorphies for cestode taxa.

\section{Applicability of the method}

Our results illustrated that the proposed protocol can successfully assemble mitochondrial sequences from genome skimming raw data of non-model organisms. We assembled the whole mitogenomes even if there were gene rearrangements, which is reinforced by the contiguity index supporting the circularization of those mitochondria.

The main advantage of this protocol is the possibility to start from a low concentration of DNA extracts (Table 1), circumventing the need for prior enrichment and can work well on samples with different levels of preservation. We believe that the critical point of this advantage lies in the library preparation kit used.

Many studies with genome skimming have used methods in which the user shears the genomic DNA through ultrasonication (Kocher et al., 2014; Richter et al., 2015) or the library prep kit requires a DNA input $\geq$ 50ng (Kocher et al., 2016; Linard et al., 2015). Such methods would probably make it impossible to sequencing some of the samples used in this study (Table 1). For mitochondrial sequencing, Nextera XT is commonly used in conjunction with enrichment methods via organelle isolation (Grandjean et al., 2017) or PCR amplification (Li et al., 2015; Foster et al., 2017), but not with genome skimming techniques. Although the Nextera XT is designed for small genomes $(\leq 5 \mathrm{Mb})$, such as genomes of bacteria and viruses, it has been used to recover mostly plastomes from plants (Burke et al., 2016).

Based on our results, we expect that the methods described here will be valuable to researcher aiming towards sequencing metazoan mitogenomes. The workflow is time-saving, and it is possible to go from DNA to the pool library in a single day. Moreover, researchers can certainly apply this protocol to other non-models organisms, in addition to old historical specimens or specimens that usually generate low concentrations of DNA from the extractions. We have demonstrated that mitochondrial genomes can be 
generated efficiently from different sequencing strategies, using Illumina MiSeq (two samples and PE reads of 300bp) and Illumina NextSeq (35 samples and PE reads of 150bp). Thus, the user can adjust the procedure costs by designing a multiplex pooling strategy that sequences the desired number of samples with suitable coverage.

\section{CONCLUSION}

The proposed method is an excellent solution to obtain low cost/large scale molecular data in biodiversity studies. Combining the adequate platform and library to the genome skimming is an innovative approach, opening a new range of possibilities of its use in obtaining molecular data from organisms with different levels of preservation. The principal advantages from our approach are: i. it requires low amount of input DNA $(0.2 n g / \mu l)$, which allows the use of organisms with ancient DNA; ii. it does not depends on specific primers and is not affected by gene rearrangement; and iii. it is time-saving and cost-effective, leading to high-quality complete sequence assemblies.

\section{ACKNOWLEDGMENTS}

We thank the Core Facility for Scientific Research-University of São Paulo (CEFAP-USP/2017) and GENIAL (Genome Investigation and Analysis Laboratory)—for the sequencing on Illumina MiSeq and NextSeq and the Museu de Zoologia da Universidade de São Paulo (MZUSP) for granting the use of computational resources (“Ace” SGI computer cluster - FAPESP \# 2012/10000-5).

\section{REFERENCES}

Alkalaeva, E. and Mikhailova, T. (2017). Reassigning stop codons via translation termination: How a few eukaryotes broke the dogma. BioEssays, 39(3):e1600213.

Altschul, S. F., Gish, W., Miller, W., Myers, E. W., and Lipman, D. J. (1990). Basic local alignment search tool. Journal of Molecular Biology, 215(3):403-410.

Andrew, S. (2010). Fastqc: A quality control tool for high throughput sequence data. Available at http://www.bioinformatics.babraham.ac.uk/projects/fastqc, version 0.7.11.

Arnason, U., Adegoke, J. A., Bodin, K., Born, E. W., Esa, Y. B., Gullberg, A., Nilsson, M., Short, R. V., Xu, X., and Janke, A. (2002). Mammalian mitogenomic relationships and the root of the eutherian tree. Proceedings of the National Academy of Sciences, 99(12):8151-8156.

Asaad, I., Lundquist, C. J., Erdmann, M. V., and Costello, M. J. (2017). Ecological criteria to identify areas for biodiversity conservation. Biological Conservation, 213:309-316.

Avise, J. C. (2012). Molecular markers, natural history and evolution. Springer Science \& Business Media.

Avise, J. C., Arnold, J., Ball, R. M., Bermingham, E., Lamb, T., Neigel, J. E., Reeb, C. A., and Saunders, N. C. (1987). Intraspecific phylogeography: The mitochondrial dna bridge between population genetics and systematics. Annual Review of Ecology and Systematics, 18(1):489-522.

Ballard, J. W. O. and Whitlock, M. C. (2004). The incomplete natural history of mitochondria. Molecular Ecology, 13(4):729-744.

Benson, D. A., Karsch-Mizrachi, I., Lipman, D. J., Ostell, J., and Sayers, E. W. (2008). Genbank. Nucleic acids research, 37(suppl_1):D26-D31.

Berger, B. A., Han, J., Sessa, E. B., Gardner, A. G., Shepherd, K. A., Ricigliano, V. A., Jabaily, R. S., and Howarth, D. G. (2017). The unexpected depths of genome-skimming data: A case study examining goodeniaceae floral symmetry genes. Applications in Plant Sciences, 5(10):e1700042.

Bernt, M., Donath, A., Jühling, F., Externbrink, F., Florentz, C., Fritzsch, G., Pütz, J., Middendorf, M., and Stadler, P. F. (2013). Mitos: Improved de novo metazoan mitochondrial genome annotation. Molecular Phylogenetics and Evolution, 69(2):313-319.

Birney, E., Clamp, M., and Durbin, R. (2004). Genewise and genomewise. Genome Research, 14(5):988995.

Brabec, J., Kostadinova, A., Scholz, T., and Littlewood, D. T. J. (2015). Complete mitochondrial genomes and nuclear ribosomal rna operons of two species of diplostomum (platyhelminthes: Trematoda): a molecular resource for taxonomy and molecular epidemiology of important fish pathogens. Parasites \& Vectors, 8(1):336. 
Brabec, J., Kuchta, R., Scholz, T., and Littlewood, D. T. J. (2016). Paralogues of nuclear ribosomal genes conceal phylogenetic signals within the invasive asian fish tapeworm lineage: evidence from next generation sequencing data. International Journal for Parasitology, 46(9):555-562.

Brooks, D. R., Thorson, T. B., and Mayes, M. A. (1981b). Freshwater stingrays (potamotrygonidae) and their helminth parasites: Testing hypothesis of evolution and coevolution. In Advances in Cladistics, proceedings of the first meeting of the Willi Hennig Society, pages 145-175. New York Botanical Garden, New York.

Burke, S. V., Wysocki, W. P., Zuloaga, F. O., Craine, J. M., Pires, J. C., Edger, P. P., Mayfield-Jones, D., Clark, L. G., Kelchner, S. A., and Duvall, M. R. (2016). Evolutionary relationships in panicoid grasses based on plastome phylogenomics (panicoideae; poaceae). BMC Plant Biology, 16(1):140.

Caira, J. N., Jensen, K., Waeschenbach, A., and Littlewood, D. (2014). An enigmatic new tapeworm, Litobothrium aenigmaticum sp. nov.(platyhelminthes: Cestoda: Litobothriidea), from the pelagic thresher shark with comments on development of known Litobothrium species. Invertebrate Systematics, 28(3):231-243.

Cameron, S. L. (2014). Insect mitochondrial genomics: Implications for evolution and phylogeny. Annual Review of Entomology, 59:95-117.

Cameron, S. L. (2014a). How to sequence and annotate insect mitochondrial genomes for systematic and comparative genomics research. Systematic Entomology, 39(3):400-411.

Cameron, S. L., Lambkin, C. L., Barker, S. C., and Whiting, M. F. (2007). A mitochondrial genome phylogeny of diptera: whole genome sequence data accurately resolve relationships over broad timescales with high precision. Systematic Entomology, 32(1):40-59.

Campbell, V. and Lapointe, F.-J. (2011). Retrieving a mitogenomic mammal tree using composite taxa. Molecular Phylogenetics and Evolution, 58(2):149-156.

Castellana, S., Vicario, S., and Saccone, C. (2011). Evolutionary patterns of the mitochondrial genome in metazoa: Exploring the role of mutation and selection in mitochondrial protein-coding genes. Genome Biology and Evolution, 3:1067-1079.

Chevreux, B., Wetter, T., and Suhai, S. (1999). Genome sequence assembly using trace signals and additional sequence information. In German Conference on Bioinformatics, volume 99, pages 45-56. Hanover, Germany.

Consortium, U. (2016). Uniprot: The universal protein knowledgebase. Nucleic Acids Research, 45(D1):D158-D169.

Corlett, R. T. (2017). A bigger toolbox: Biotechnology in biodiversity conservation. Trends in Biotechnology, 35(1):55-65.

Dabney, J., Knapp, M., Glocke, I., Gansauge, M.-T., Weihmann, A., Nickel, B., Valdiosera, C., García, N., Pääbo, S., Arsuaga, J.-L., and Matthias, M. (2013). Complete mitochondrial genome sequence of a middle pleistocene cave bear reconstructed from ultrashort dna fragments. Proceedings of the National Academy of Sciences, 110(39):15758-15763.

Dick, C. W., Graciolli, G., and Guerrero, R. (2016). Family streblidae. Zootaxa, 4122(1):784-802.

Dick, C. W. and Patterson, B. D. (2006). Bat flies: Obligate ectoparasites of bats. In Micromammals and macroparasites, pages 179-194. Springer.

Dittmar, K., Porter, M. L., Murray, S., and Whiting, M. F. (2006). Molecular phylogenetic analysis of nycteribiid and streblid bat flies (diptera: Brachycera, calyptratae): Implications for host associations and phylogeographic origins. Molecular Phylogenetics and Evolution, 38(1):155-170.

do Amaral, F. R., Neves, L. G., Resende Jr, M. F., Mobili, F., Miyaki, C. Y., Pellegrino, K. C., and Biondo, C. (2015). Ultraconserved elements sequencing as a low-cost source of complete mitochondrial genomes and microsatellite markers in non-model amniotes. Plos One, 10(9):e0138446.

Dodsworth, S. (2015). Genome skimming for next-generation biodiversity analysis. Trends in Plant Science, 20(9):525-527.

Egger, B., Bachmann, L., and Fromm, B. (2017). Atp8 is in the ground pattern of flatworm mitochondrial genomes. BMC Genomics, 18(1):414.

Ekblom, R. and Galindo, J. (2011). Applications of next generation sequencing in molecular ecology of non-model organisms. Heredity, 107(1):1.

Foster, P. G., de Oliveira, T. M. P., Bergo, E. S., Conn, J. E., Sant'Ana, D. C., Nagaki, S. S., Nihei, S., Lamas, C. E., González, C., Moreira, C. C., and Sallum, M. A. M. (2017). Phylogeny of anophelinae using mitochondrial protein coding genes. Royal Society Open Science, 4(11):170758. 
Goeze, J. A. E. (1782). Versuch einer Naturgeschichte der Eingeweidewürmer thierischer Körper: Mit 44 Kupfertafeln. Crusius.

Grandjean, F., Tan, M. H., Gan, H. M., Lee, Y. P., Kawai, T., Distefano, R. J., Blaha, M., Roles, A. J., and Austin, C. M. (2017). Rapid recovery of nuclear and mitochondrial genes by genome skimming from northern hemisphere freshwater crayfish. Zoologica Scripta, 46(6):718-728.

Guo, A. (2015). The complete mitochondrial genome of Anoplocephala perfoliata, the first representative for the family anoplocephalidae. Parasites \& Vectors, 8(1):549.

Guo, A. (2016). The complete mitochondrial genome of the tapeworm Cladotaenia vulturi (cestoda: Paruterinidae): Gene arrangement and phylogenetic relationships with other cestodes. Parasites \& Vectors, 9(1):475.

Hahn, C., Bachmann, L., and Chevreux, B. (2013). Reconstructing mitochondrial genomes directly from genomic next-generation sequencing reads-a baiting and iterative mapping approach. Nucleic Acids Research, 41(13):e129-e129.

Hartikainen, H., Bass, D., Briscoe, A. G., Knipe, H., Green, A. J., and Okamura, B. (2016). Assessing myxozoan presence and diversity using environmental dna. International Journal for Parasitology, 46(12):781-792.

Heintzman, P. D., Elias, S. A., Moore, K., Paszkiewicz, K., and Barnes, I. (2014). Characterizing dna preservation in degraded specimens of Amara alpina (carabidae: Coleoptera). Molecular Ecology Resources, 14(3):606-615.

Hu, M., Gasser, R., Chilton, N., and Beveridge, I. (2005). Genetic variation in the mitochondrial cytochrome c oxidase subunit 1 within three species of progamotaenia (cestoda: Anoplocephalidae) from macropodid marsupials. Parasitology, 130(1):117-129.

Kanda, K., Pflug, J. M., Sproul, J. S., Dasenko, M. A., and Maddison, D. R. (2015). Successful recovery of nuclear protein-coding genes from small insects in museums using illumina sequencing. Plos One, 10(12): 0143929.

Kang, S., Tice, A. K., Spiegel, F. W., Silberman, J. D., Pánek, T., Čepička, I., Kostka, M., Kosakyan, A., Alcântara, D. M., Roger, A. J., Shadwick, L. L., Smirnov, A., Kudryavtsev, A., Lahr, D. J., and Brown, M. W. (2017). Between a pod and a hard test: The deep evolution of amoebae. Molecular Biology and Evolution, 34(9):2258-2270.

Kocher, A., Gantier, J.-C., Holota, H., Jeziorski, C., Coissac, E., Bañuls, A.-L., Girod, R., Gaborit, P., and Murienne, J. (2016). Complete mitochondrial genome of Lutzomyia (Nyssomyia) umbratilis (diptera: Psychodidae), the main vector of Leishmania guyanensis. Mitochondrial DNA Part A, 27(6):4219-4221.

Kocher, A., Kamilari, M., Lhuillier, E., Coissac, E., Péneau, J., Chave, J., and Murienne, J. (2014). Shotgun assembly of the assassin bug Brontostoma colossus mitochondrial genome (heteroptera, reduviidae). Gene, 552(1):184-194.

Langmead, B. and Salzberg, S. L. (2012). Fast gapped-read alignment with bowtie 2. Nature Methods, 9(4):357.

Laslett, D. and Canbäck, B. (2007). Arwen: A program to detect trna genes in metazoan mitochondrial nucleotide sequences. Bioinformatics, 24(2):172-175.

Le, T. H., Blair, D., Agatsuma, T., Humair, P.-F., Campbell, N. J., Iwagami, M., Littlewood, D. T. J., Peacock, B., Johnston, D. A., Bartley, J., Rollinson, D., Herniou, E., Zarlenga, S., and McManus, D. (2000). Phylogenies inferred from mitochondrial gene orders-a cautionary tale from the parasitic flatworms. Molecular Biology and Evolution, 17(7):1123-1125.

Le, T. H., Blair, D., and McManus, D. P. (2002). Mitochondrial genomes of parasitic flatworms. Trends in Parasitology, 18(5):206-213.

Li, W. X., Zhang, D., Boyce, K., Xi, B. W., Zou, H., Wu, S. G., Li, M., and Wang, G. T. (2017). The complete mitochondrial dna of three monozoic tapeworms in the caryophyllidea: A mitogenomic perspective on the phylogeny of eucestodes. Parasites \& Vectors, 10(1):314.

Li, X., Ding, S., Cameron, S. L., Kang, Z., Wang, Y., and Yang, D. (2015). The first mitochondrial genome of the sepsid fly Nemopoda mamaevi ozerov, 1997 (diptera: Sciomyzoidea: Sepsidae), with mitochondrial genome phylogeny of Cyclorrhapha. Plos One, 10(3):1-26.

Linard, B., Arribas, P., Andújar, C., Crampton-Platt, A., and Vogler, A. (2016). Lessons from genome skimming of arthropod-preserving ethanol. Molecular Ecology Resources, 16(6):1365-1377.

Linard, B., Crampton-Platt, A., Gillett, C. P., Timmermans, M. J., and Vogler, A. P. (2015). Metagenome skimming of insect specimen pools: Potential for comparative genomics. Genome Biology and 
Evolution, 7(6):1474-1489.

Littlewood, D., Waeschenbach, A., and Nikolov, P. (2008). In search of mitochondrial markers for resolving the phylogeny of cyclophyllidean tapeworms (platyhelminthes, cestoda) - a test study with davaineidae. Acta Parasitologica, 53(2):133-144.

Liu, Z.-Q., Kuermanali, N., Li, Z., Chen, S.-J., Wang, Y.-Z., Tao, H., and Chen, C.-F. (2017). The complete mitochondrial genome of the parasitic sheep ked Melophagus ovinus (diptera: Hippoboscidae). Mitochondrial DNA Part B, 2(2):432-434.

Lowe, T. M. and Eddy, S. R. (1997). trnascan-se: A program for improved detection of transfer rna genes in genomic sequence. Nucleic Acids Research, 25(5):955.

Machado, D., Lyra, M., and Grant, T. (2016). Mitogenome assembly from genomic multiplex libraries: Comparison of strategies and novel mitogenomes for five species of frogs. Molecular Ecology Resources, 16(3):686-693.

Machado, D. J., Janies, D., Brouwer, C., and Grant, T. (2018). A new strategy to infer circularity applied to four new complete frog mitogenomes. Ecology and evolution, 8(8):4011-4018.

Maddison, D. R. and Cooper, K. W. (2014). Species delimitation in the ground beetle subgenus Liocosmius (coleoptera: Carabidae: Bembidion), including standard and next-generation sequencing of museum specimens. Zoological Journal of the Linnean Society, 172(4):741-770.

Maldonado, L. L., Assis, J., Araújo, F. M. G., Salim, A. C., Macchiaroli, N., Cucher, M., Camicia, F., Fox, A., Rosenzvit, M., Oliveira, G., and Kamenetzky, L. (2017). The Echinococcus canadensis (g7) genome: A key knowledge of parasitic platyhelminth human diseases. BMC Genomics, 18(1):204.

Marques, F. P. and Caira, J. N. (2016). Pararhinebothroides - neither the sister-taxon of Rhinebothroides nor a valid genus. The Journal of Parasitology, 102(2):249-259.

Marques, F. P. and Reyda, F. B. (2015). Rhinebothrium jaimei sp. n. (eucestoda: Rhinebothriidea: Rhinebothriidae): A new species from neotropical freshwater stingrays (potamotrygonidae). Folia Parasitologica, 62:1.

Matos-Maraví, P., Ritter, C. D., Barnes, C. J., Nielsen, M., Olsson, U., Wahlberg, N., Marquina, D., Sääksjärvi, I., and Antonelli, A. (2018). Biodiversity seen through the perspective of insects: 10 simple rules on methodological choices, common challenges, and experimental design for genomic studies. PeerJ PrePrints.

McCormack, J. E., Rodríguez-Gómez, F., Tsai, W. L., and Faircloth, B. C. (2017). Transforming museum specimens into genomic resources. The Extended Specimen: Emerging Frontiers in Collections Based Ornithological Research, pages 143-156.

McCormack, J. E., Tsai, W. L., and Faircloth, B. C. (2016). Sequence capture of ultraconserved elements from bird museum specimens. Molecular Ecology Resources, 16(5):1189-1203.

Nakao, M., Sako, Y., and Ito, A. (2003). The mitochondrial genome of the tapeworm Taenia solium: A finding of the abbreviated stop codon u. Journal of Parasitology, 89(3):633-635.

Narayanan Kutty, S., Meusemann, K., Bayless, K. M., Marinho, M. A., Pont, A. C., Zhou, X., Misof, B., Wiegmann, B. M., Yeates, D., Cerretti, P., Meier, R., and Pape, T. (2019). Phylogenomic analysis of calyptratae: resolving the phylogenetic relationships within a major radiation of diptera. Cladistics.

Nelson, L. A., Lambkin, C. L., Batterham, P., Wallman, J. F., Dowton, M., Whiting, M. F., Yeates, D. K., and Cameron, S. L. (2012). Beyond barcoding: A mitochondrial genomics approach to molecular phylogenetics and diagnostics of blowflies (diptera: Calliphoridae). Gene, 511(2):131-142.

Oliveira, U., Soares-Filho, B. S., Paglia, A. P., Brescovit, A. D., Carvalho, C. J., Silva, D. P., Rezende, D. T., Leite, F. S. F., Batista, J. A. N., Barbosa, J. P. P. P., Stehmann, J. R., Ascher, J. S., de Vasconcelos, M. F., De Marco, P., Löwenberg-Neto, P., Ferro, V. G., and Santos, A. J. (2017). Biodiversity conservation gaps in the brazilian protected areas. Scientific Reports, 7(1):9141.

Pacheco, M. A., Battistuzzi, F. U., Lentino, M., Aguilar, R. F., Kumar, S., and Escalante, A. A. (2011). Evolution of modern birds revealed by mitogenomics: Timing the radiation and origin of major orders. Molecular Biology and Evolution, 28(6):1927-1942.

Park, J.-K., Kim, K.-H., Kang, S., Jeon, H., Kim, J.-H., Littlewood, D., and Eom, K. (2006). Characterization of the mitochondrial genome of Diphyllobothrium latum (cestoda: Pseudophyllidea) - implications for the phylogeny of eucestodes. Parasitology, 134(5):749-759.

Petersen, F. T., Meier, R., Kutty, S. N., and Wiegmann, B. M. (2007). The phylogeny and evolution of host choice in the hippoboscoidea (diptera) as reconstructed using four molecular markers. Molecular Phylogenetics and Evolution, 45(1):111-122. 
Pleijel, F., Jondelius, U., Norlinder, E., Nygren, A., Oxelman, B., Schander, C., Sundberg, P., and Thollesson, M. (2008). Phylogenies without roots? A plea for the use of vouchers in molecular phylogenetic studies. Molecular Phylogenetics Evolution, 48:369-371.

Porter, T. M. and Hajibabaei, M. (2018). Scaling up: A guide to high throughput genomic approaches for biodiversity analysis. Molecular Ecology, 27(2):313-338.

Pu, D.-q., Liu, H.-1., Gong, Y.-y., Ji, P.-c., Li, Y.-j., Mou, F.-s., and Wei, S.-j. (2017). Mitochondrial genomes of the hoverflies Episyrphus balteatus and Eupeodes corollae (diptera: Syrphidae), with a phylogenetic analysis of Muscomorpha. Scientific Reports, 7:e44300.

Richter, S., Schwarz, F., Hering, L., Böggemann, M., and Bleidorn, C. (2015). The utility of genome skimming for phylogenomic analyses as demonstrated for glycerid relationships (annelida, glyceridae). Genome Biology and Evolution, 7(12):3443-3462.

Ruhnke, T. R., Caira, J. N., and Cox, A. (2015). The cestode order rhinebothriidea no longer family-less: A molecular phylogenetic investigation with erection of two new families and description of eight new species of Anthocephalum. Zootaxa, 3904(1):51-81.

Ruhnke, T. R., Reyda, F. B., and Marques, F. P. L. (2017). Rhinebothriidea healy, caira, jensen, webster \& littlewood, 2009. In Caira., J. N. and Jensen, K., editors, Planetary Biodiversity Inventory (PBI): Tapeworms from vertebrate bowels of the earth (2008-2017)., pages 327-348. University of Kansas Natural History Museum Special Publication. Vol. 25.

Schattner, P., Brooks, A. N., and Lowe, T. M. (2005). The trnascan-se, snoscan and snogps web servers for the detection of trnas and snornas. Nucleic Acids Research, 33(suppl_2):W686-W689.

Staats, M., Erkens, R. H., van de Vossenberg, B., Wieringa, J. J., Kraaijeveld, K., Stielow, B., Geml, J., Richardson, J. E., and Bakker, F. T. (2013). Genomic treasure troves: Complete genome sequencing of herbarium and insect museum specimens. Plos One, 8(7):e69189.

Tan, M. H., Gan, H. M., Lee, Y. P., Poore, G. C., and Austin, C. M. (2017). Digging deeper: New gene order rearrangements and distinct patterns of codons usage in mitochondrial genomes among shrimps from the axiidea, gebiidea and caridea (crustacea: Decapoda). PeerJ, 5:e2982.

Tello, S., Stevens, R. D., and Dick, C. W. (2008). Patterns of species co-occurrence and density compensation: A test for interspecific competition in bat ectoparasite infracommunities. Oikos, 117(5):693-702.

Tilak, M.-K., Justy, F., Debiais-Thibaud, M., Botero-Castro, F., Delsuc, F., and Douzery, E. J. (2015). A cost-effective straightforward protocol for shotgun illumina libraries designed to assemble complete mitogenomes from non-model species. Conservation Genetics Resources, 7(1):37-40.

Timmermans, M. J. T. N., Viberg, C., Martin, G., Hopkins, K., and Vogler, A. P. (2016). Rapid assembly of taxonomically validated mitochondrial genomes from historical insect collections. Biological Journal of the Linnean Society, 117(1):83-95.

Trevisan, B., Primon, J. F., and Marques, F. P. (2017). Systematics and diversification of Anindobothrium marques, brooks \& lasso, 2001 (eucestoda: Rhinebothriidea). Plos One, 12(9):e0184632.

Twyford, A. D. and Ness, R. W. (2017). Strategies for complete plastid genome sequencing. Molecular Ecology Resources, 17(5):858-868.

Vanhove, M. P., Briscoe, A. G., Jorissen, M. W., Littlewood, D. T. J., and Huyse, T. (2018). The first next-generation sequencing approach to the mitochondrial phylogeny of african monogenean parasites (platyhelminthes: Gyrodactylidae and dactylogyridae). BMC Genomics, 19(1):520.

Vellend, M. (2017). The biodiversity conservation paradox. American Scientist, 105(2):94.

von Nickisch-Rosenegk, M., Lucius, R., and Loos-Frank, B. (1999). Contributions to the phylogeny of the cyclophyllidea (cestoda) inferred from mitochondrial 12s rdna. Journal of Molecular Evolution, 48(5):586-596.

Waeschenbach, A., Webster, B., and Littlewood, D. (2012). Adding resolution to ordinal level relationships of tapeworms (platyhelminthes: Cestoda) with large fragments of mtdna. Molecular Phylogenetics and Evolution, 63(3):834-847.

Wenzel, R. L., Tipton, V. J., and Kiewlicz, A. (1966). The streblid batflies of Panama (Diptera Calypterae: Streblidae). Field Museum of Natural History.

Wickström, L. M., Haukisalmi, V., Varis, S., Hantula, J., and Henttonen, H. (2005). Molecular phylogeny and systematics of anoplocephaline cestodes in rodents and lagomorphs. Systematic Parasitology, 62(2):83-99.

Yan, D., Tang, Y., Xue, X., Wang, M., Liu, F., and Fan, J. (2012). The complete mitochondrial genome 
sequence of the western flower thrips frankliniella occidentalis (thysanoptera: Thripidae) contains triplicate putative control regions. Gene, 506(1):117-124.

Yang, X., Liu, D., Liu, F., Wu, J., Zou, J., Xiao, X., Zhao, F., and Zhu, B. (2013). Htqc: A fast quality control toolkit for illumina sequencing data. BMC Bioinformatics, 14(1):33.

Yuan, S., Xia, Y., Zheng, Y., and Zeng, X. (2016). Next-generation sequencing of mixed genomic dna allows efficient assembly of rearranged mitochondrial genomes in amolops chunganensis and quasipaa boulengeri. PeerJ, 4:e2786.

Zarowiecki, M., Huyse, T., and Littlewood, D. (2007). Making the most of mitochondrial genomesmarkers for phylogeny, molecular ecology and barcodes in Schistosoma (platyhelminthes: Digenea). International Journal for Parasitology, 37(12):1401-1418.

Zehnder, M. and Mariaux, J. (1999). Molecular systematic analysis of the order proteocephalidea (eucestoda) based on mitochondrial and nuclear rdna sequences1. International Journal for Parasitology, 29(11):1841-1852.

Zhang, X., Duan, J. Y., Shi, Y. L., Jiang, P., Zeng, D. J., Wang, Z. Q., and Cui, J. (2017). Comparative mitochondrial genomics among Spirometra (cestoda: Diphyllobothriidae) and the molecular phylogeny of related tapeworms. Molecular Phylogenetics and Evolution, 117:75-82.

Zhao, G., Wang, H., Jia, Y., Zhao, W., Hu, X., Yu, S., and Liu, G. (2016). The complete mitochondrial genome of Pseudanoplocephala crawfordi and a comparison with closely related cestode species. Journal of Helminthology, 90(5):588-595. 